\title{
I maging systolic and diastolic heart murmurs in hypertrophic cardiomyopathy
}

\author{
Andrea Barison ${ }^{1,2}$, Giancarlo Todiere², Pier Giorgio Masci², Giovanni D Aquaro² \\ 1. Scuola Superiore Sant'Anna, Pisa, Italy. 2. Fondazione “G.Monasterio" CNR - Regione Toscana, Pisa, Italy \\ Correspondence: Andrea Barison. Address: Fondazione “G. Monasterio" CNR - Regione Toscana, Via Moruzzi, 1 - 56124 \\ Pisa, Italy. Email: barison@sssup.it
}

Received: January 10, 2014

Accepted: February 8, 2014

Online Published: February 21, 2014

DOI : $10.5430 /$ ijdi.v1n1p1

URL: http://dx.doi.org/10.5430/ijdi.v1n1p1

\begin{abstract}
Muscular obstruction in hypertrophic cardiomyopathy can occur not only in systole, but also in diastole. Here we report a case of hypertrophic cardiomyopathy in a middle-aged lady: echocardiography and cardiovascular magnetic resonance confirmed the diagnosis of hypertrophic cardiomyopathy with significant systolic and diastolic midventricular obstruction, corresponding to the murmurs audible in mid-systole and early-diastole respectively. Moreover, the mid-systolic murmur was clearly distinguishable from the mitral regurgitation murmur, which was audible throughout systole (pan-systolic) and likely related to systolic anterior motion of the mitral chords; similarly, the early-diastolic heart murmur due to diastolic obstruction was better audible at the apex and clearly distinguishable from common diastolic murmurs due to aortic regurgitation.

Echocardiography (with Doppler recordings) and magnetic resonance (with turbulence flows at steady state free precession imaging) allowed a detailed visualization of each audible murmur (systolic obstruction, mitral regurgitation, diastolic obstruction). In particular, while systolic obstruction has been extensively studied in hypertrophic cardiomyopathy, only very few reports about diastolic obstruction exist, describing either a normodirected midventricular diastolic obstruction (forward flow) similar to this case or a paradoxical midventricular diastolic obstruction (backward flow) in patients with an apical aneurysm emptying during early diastole into the left ventricular base through a narrow midventricular channel. Whether diastolic obstruction implies worse prognosis or requires specific treatment, deserves further research.
\end{abstract}

\section{Keywords}

Hypertrophic cardiomyopathy, Cardiac murmurs, Systolic obstruction, Diastolic obstruction, Magnetic resonance, Echocardiography

\section{I ntroduction}

Systolic muscular obstruction has been described in one third of hypertrophic cardiomyopathy patients at rest, and in another third after exercise stress ${ }^{[1]}$. Midventricular obstruction, in particular, is an infrequent phenotype, associated with adverse prognosis ${ }^{[2]}$. On the other hand, muscular diastolic obstruction has been reported rarely in hypertrophic cardiomyopathy. 


\section{Case presentation}

A 52-year-old lady with family history of hypertrophic cardiomyopathy and premature sudden death was admitted for breathlessness. At physical examination she presented diffuse lung crackles, a harsh mid-systolic heart murmur, a regurgitant pan-systolic murmur, as well as an apical early-diastolic murmur. Her ECG showed sinus rhythm with diffuse hypertrophy, and transthoracic echocardiography (iE33, Philips, The Netherlands) showed asymmetrical septal hypertrophy (see Figure 1) with midventricular systolic obstruction (mean gradient $40 \mathrm{mmHg}$ at rest, rising to $50 \mathrm{mmHg}$ during Valsalva manoeuvre) (see Figure 1D), systolic anterior motion of the mitral chords with moderate mitral regurgitation, as well as early-diastolic midventricular obstruction (mean gradient $12 \mathrm{mmHg}$ at rest) (see Figure 1E). A normal valve opening (see Figure 1A,B) and "pseudonormal" transmitral velocities at pulsed-wave Doppler (see Figure 1C) ruled out any primary mitral disease, while midventricular flow aliasing in both systole in diastole at pulsed-wave Doppler confirmed the systo-diastolic midventricular obstruction.

Cardiovascular magnetic resonance (CVi 1.5T, GE-Healthcare, Milwaukee-USA) (see Figure 2) confirmed the hypertrophic cardiomyopathy with flow turbulence due to mitral regurgitation (see Figure 2A, early systole), midventricular (muscular) and subaortic (chordal) systolic obstruction (see Figure 2B, mid systole) as well as midventricular early diastolic obstruction (see Figure 2C, early diastole). After contrast injection, magnetic resonance disclosed also the presence of midventricular late gadolinium enhancement (see Figure 3).

During hospitalization the patient underwent genetic counselling and testing, which disclosed a familiar form of obstructive hypertrophic cardiomyopathy due to a double mutation in the myosin binding protein C-3 gene. After medical therapy optimization (verapamil $360 \mathrm{mg} /$ day) and implantation of an intracardiac cardioverter defibrillator, she remained asymptomatic at 12-month follow-up, with echocardiographic reduction of the midventricular obstruction.

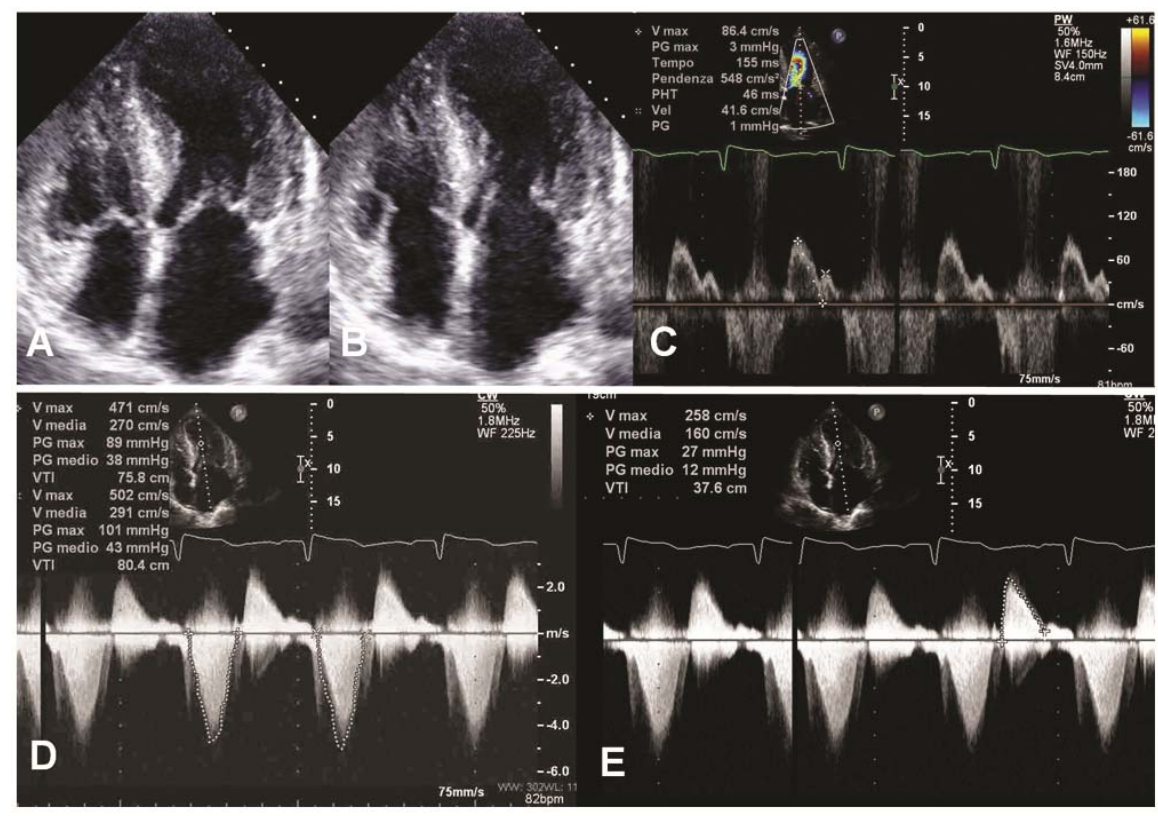

Figure 1. Transthoracic echocardiographic bidimensional and Doppler imaging.Transthoracic echocardiography apical four-chamber view showing midventricular hypertrophic cardiomyopathy in mid-diastole (A) and end-diastole (B). Pulsed-wave Doppler recording at mitral leaflets level (C) showed normal transmitral velocities with a "pseudonormal" filling pattern. Continuous-wave Doppler echocardiography along the left ventricular longitudinal axis showed: (D) a late-peaking systolic flow acceleration, representing the midventricular systolic obstruction and appearing superimposed on the early-peaking systolic flow of mitral regurgitation; (E) an early-peaking diastolic flow acceleration, representing the midventricular early-diastolic flow obstruction. 


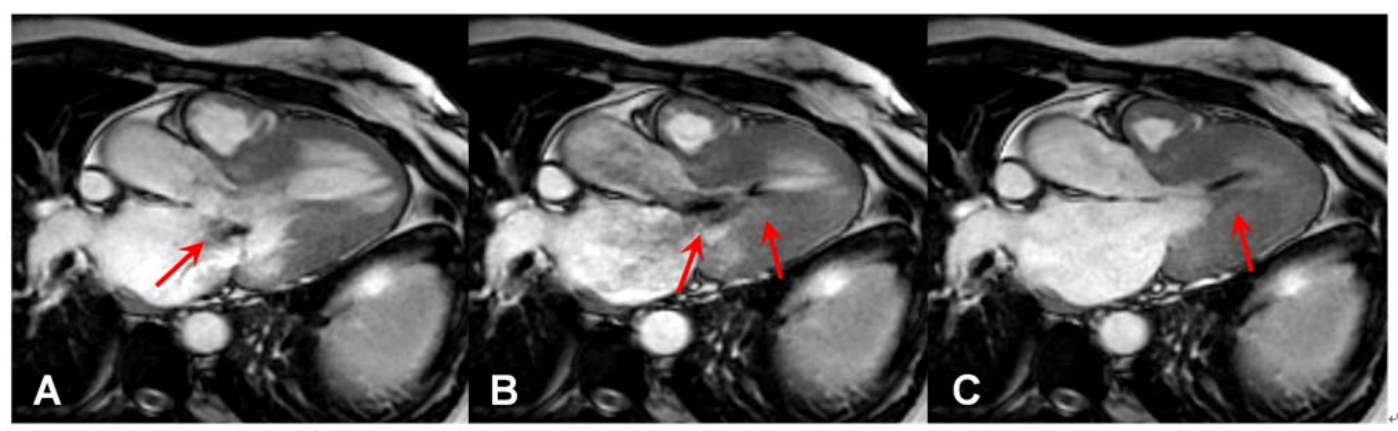

Figure 2. Cardiovascular magnetic resonance cine imaging. Balanced steady-state free precession three-chamber view of the left ventricle, showing asymmetrical hypertrophy with flow turbulence (arrows) due to mitral regurgitation (A, early systole), midventricular (muscular) and subaortic (chordal) obstruction during mid systole (B), as well as midventricular obstruction during early diastole (C). Sequence parameters were: field-of-view: 360mm, thickness/spacing: 8/0mm; repetition/echo time: 3.5/1.5ms, flip angle: $60^{\circ}$, matrix: 224×224, phases: 30; views-per-segment: 12 .

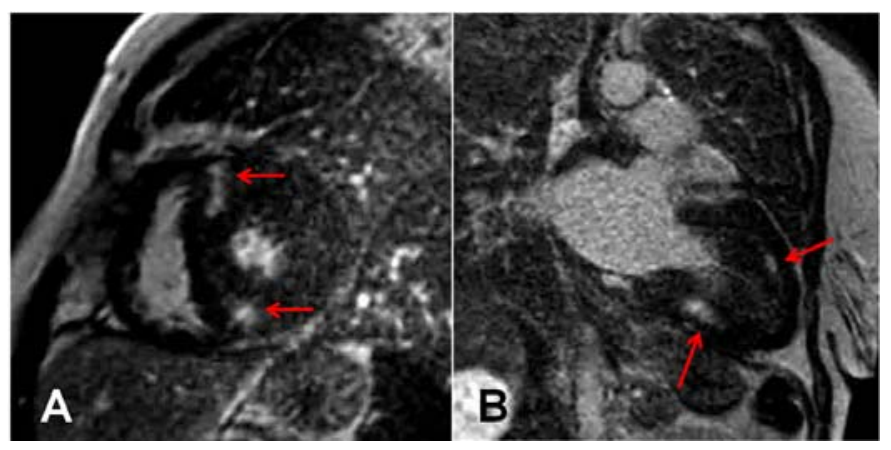

Figure 3. Cardiovascular magnetic resonance late gadolinium enhancement imaging. Segmented T1-weighted inversionrecovery gradient-echo imaging acquired $10 \mathrm{~min}$ after gadolinium-contrast injection $(0.2 \mathrm{mmmol} / \mathrm{Kg}$ gadoteri acid, DOTAREM, Guerbet, France), showing two areas of late enhancement (red arrows) in the interventricular septum in short- (A) and long (B) axis view, representing midwall myocardial fibrosis. Sequences parameters were: field-of-view: 360mm, thickness/spacing: 8/0mm; repetition/echo time: 4.6/1.3ms, flip angle: $20^{\circ}$, matrix: $224 \times 192$, phases: 30 ; views-per-segment: 12.

\section{Discussion}

Muscular diastolic obstruction has been reported very rarely in hypertrophic cardiomyopathy, mainly as a paradoxical midventricular diastolic flow running back from an apical aneurism to the left ventricular base through a narrow midventricular channel ${ }^{[3,4]}$. In particular, previous reports described a high-pressure sequestered apical aneurism with a dyssynchronous delayed movement, filling during early systole (isovolumic ventricular contraction) and emptying during early diastole. Differently, in our case there was a normally directed diastolic flow, i.e. from the left ventricular base to the apex. Forward flow obstruction occurred soon after mitral valve opening and regressed soon after midventricular relaxation. The most likely mechanism could be the prolongation of left ventricular cavity obliteration well into early diastole, but left ventricular apical suction during early diastole might contribute to a midventricular "Venturi" effect. As far as the early-diastolic cardiac murmur was much better appreciated at the apex, it could be distinguished from common diastolic murmurs due to aortic regurgitation. Diastolic obstruction is likely related to more advanced disease and requires 
enhanced therapeutic effort and closer follow-up, but whether it implies worse prognosis or requires other specific treatment is still unknown.

\section{References}

[1] Maron MS, Olivotto I, Zenovich AG, Link MS, Pandian NG, Kuvin JT, et al. Hypertrophic cardiomyopathy is predominantly a disease of left ventricular outflow tract obstruction. Circulation. 2006; 114: 2232-39.

http://dx.doi.org/10.1161/CIRCULATIONAHA.106.644682

[2] Minami Y, Kajimoto K, Terajima Y, Yashiro B, Okayama D, Haruki S, et al. Clinical implications of midventricular obstruction in patients with hypertrophic cardiomyopathy. J Am Coll Cardiol. 2011; 57: 2346-55.

http://dx.doi.org/10.1016/j.jacc.2011.02.033

[3] Zoghbi WA, Haichin RN, Quinones MA Mid-cavity obstruction in apical hypertrophy: Doppler evidence of diastolic intraventricular gradient with higher apical pressure. Am Heart J. 1988; 116: 1469-74. http://dx.doi.org/10.1016/0002-8703(88)90730-2

[4] Nakamura T, Matsubara K, Furukawa K, Azuma A, Sugihara H, Katsume H, et al. Diastolic paradoxic jet flow in patients with hypertrophic cardiomyopathy: evidence of concealed apical asynergy with cavity obliteration. J Am Coll Cardiol. 1992; 19: 516-24. http://dx.doi.org/10.1016/S0735-1097(10)80264-5 